\title{
Producir textos desde las ciencias sociales: una posibilidad de interdisciplinariedad (1)
}

To produce texts from the social sciences: an interdisciplinary possibility $t(1)$

\author{
Janeth Patricia Barbosa Verjel \\ Especialista de la Recreación Ecológica \\ Fundación Universitaria los Libertadores \\ Maestría (T) en Educación \\ Universidad Autónoma de Bucaramanga \\ Docente de la Institución Educativa La Salle \\ Ocaña - Norte de Santander - Colombia. \\ jbarbosa317@unab.edu.co \\ janethpaty@hotmail.es
}

Socorro Astrid Portillo Castellanos

Magíster en Gestión de la Calidad de la Educación Superior

Universidad de Pamplona

Docente asociado

Universidad Autónoma de Bucaramanga

sportilla2@ unab.edu.co

Artículo recibido el 10 de abril del 2016

Aprobado el 02 de mayo del 2016(2)

\section{Resumen}

"Saber leer y escribir constituye una capacidad necesaria en sí misma, y es la base de otras aptitudes vitales... Es preciso adelantar acciones desde la escuela que favorezcan las competencias comunicativas...” UNESCO, plan sectorial (MEN, 2011: 32). 
Esta investigación tiene como objetivo promover la producción textual en ciencias sociales, seleccionando 41 estudiantes de sexto, Colegio la Salle de Ocaña. Usando la investigación-acción, encuestas, prueba diagnóstica y rúbricas. Logrando el $100 \%$ de participación, poemario-astronómico, creación de la escuela de escritores, selección de cuentos por el MEN para publicar, elaboración de atlas y aceptación de la investigación como propuesta para secundaria.

Palabras Clave: Competencias comunicativas, producción textual, ciencias sociales, educación secundaria.

\begin{abstract}
"Knowing how to read and writing establishes a necessary capacity by itself, and it's the foundation of other vital aptitudes... It is necessary to advance some actions UNESCO, plan sectorial (MEN, 2011: 32).
\end{abstract}

This research aims to promote textual production in Social Sciences, selecting forty-one sixth graders from La Salle school in Ocaña. Employing Investigation-Action, surveys, diagnostic test and rubrics, reaching one-hundred percent participation, astronomical poetry, establishing school of writers, selection of tales sent to MEN (Ministry of National Education) to be published, working out atlas and acceptance of the research as a proposal for high school.

Key words: Communicative competences, writing, social sciences, high school education.

\title{
Introducción
}

"El proceso de escribir me recuerda los preparativos para una fiesta. No sabes a cuánta gente invitar, ni qué menú escoger, ni qué mantel poner... Ensucias ollas, platos, vasos, cucharas y cazos. Derramas aceite, lo pisoteas, resbalas, vas por los suelos, sueltas cuatro palabrotas, maldices el día en que se te ocurrió la feliz idea de complicarte la existencia. Finalmente, llegan los invitados y todo está limpio y reluciente, como si nada hubiera pasado. Los amigos te felicitan por el banquete y tú sueltas una de esas frases matadoras: «Nada..., total media hora... ¡Todo lo ha hecho el horno!» (Cassany, 1993: 12)

La investigación donde se origina este artículo surge a partir del siguiente interrogante ¿Cómo promover la producción textual en estudiantes de sexto grado en el área de ciencias sociales?, como propuesta al proceso de formación de la Maestría en Educación, de la Universidad Autónoma de Bucaramanga, dentro del programa Becas por la excelencia Educativa condonada con el Ministerio de Educación Nacional. Para lo cual fue necesario realizar un recorrido de diversos escenarios bibliográficos donde se destaca la importancia de los 
procesos de producción textual en el aula permitiendo obtener paso a paso herramientas de aplicación. Domínguez (2012) reafirma la interdisciplinariedad que debe manejar el docente para estar en condiciones de diseñar situaciones didácticas coherentes con los fines educativos en particular con las habilidades comunicativas. Permite tener claro no enfatizar la producción solo con fines gramaticales, sintácticos, ortográficos o verificación solo de aprendizajes. Se debe validar la intención de comunicación y permitir la expresión de sentimientos.

La reflexión constante sobre las prácticas pedagógicas en torno a los procesos de producción textual es un factor primordial en el desarrollo de competencias comunicativas, identificando aspectos claves como la fluidez, creatividad, placer y el manejo de las fuentes. (Chávez, Murata \& Uechara, 2012)

La encuesta curricular realizada por el ICFES en el 2008 a docentes para indagar sobre las prácticas pedagógicas en trabajos de escritura, presenta un resumen de las principales falencias encontradas. (MEN, 2011: 4). Lo cual motivó a buscar posibles soluciones mediante la organización de unidades didácticas en el área de ciencias sociales para estudiantes de sexto grado, involucrando de manera creativa la producción de diferentes tipos de textos, incluyendo poemas, los cuales posteriormente fueron recopilados en pequeños libros que fueron distribuidos entre los mismos niños. La propuesta apunta a los tres objetivos planteados en el Plan Nacional de Lectura y Escritura de Educación Inicial, relacionados con los logros de la enseñanza de la escritura: escribir para expresar la subjetividad, escribir para generar conocimiento y construirse como autores. Escribir para ejercer la ciudadanía. (MEN, 2011). Es así como se plantea el objetivo general de promover la producción textual en estudiantes de sexto grado del Colegio La Salle de Ocaña en el área de ciencias sociales mediante la utilización de diversas estrategias.

Es necesario retomar teorías aceptadas en relación a la producción textual, para permitir al docente la comprensión de este proceso y la reflexión sobre sus prácticas pedagógica desde el área de ciencias sociales. Así puede seleccionar estrategias adecuadas:

Teoría de Haye, (1996) citado por Álvarez \& Ramírez, (2006) quien incorpora elementos relevantes como la memoria, la motivación y procesos cognitivos de interpretación y reflexión. Desde esta mirada se pude analizar que es posible partir de las preguntas generadoras, las cuales favorecen la motivación y los procesos cognitivos a través de la indagación para solucionarlas.

Teoría de Nystrand (1982) citado por Álvarez \& Ramírez, (2006). Establece la necesidad de indagar por los objetivos de quien escribe y resaltar la manera de hablar del lector, la necesidad sociolingüística y etnográfica de las comunidades. Es así como en el proceso de producción textual enumera los pasos de invención, planificación, estilo, memoria y entrega. Por otra parte menciona el papel que juegan los estados de ánimo del escritor, el miedo a cometer errores y a dejar la hoja en blanco, lo cual sugiere el manejo de inteligencia 
emocional, (Goleman, 1996).Con esto se evidencia la relación directa que existe entre las ciencias sociales y la posibilidad de ejecutar a través de ella procesos de producción textual, porque permite el conocimiento de las diversas culturas, el manejo de las características propias del contexto. De estrategias que faciliten el arte de escribir, buscando un estilo propio en cada estudiante. Es necesario mencionar que todas esas acciones generan el despliegue del imaginario, minimizando el temor a no poder lograr el cometido de escribir algo. De igual manera la producción textual en sí, permite ser una herramienta de resiliencia desde las ciencias sociales, canalizando los temores, frustraciones y desdichas de los niños y niñas afectados por el desplazamiento y la violencia del país y específicamente de la zona del Catatumbo.

\section{Metodología}

La metodología adoptada es investigación acción, destacando las ideas de John Elliott quien formula un modelo sobre la base del modelo cíclico de Lewin, destacando tres momentos: "elaborar el plan, ponerlo en marcha y evaluarlo; rectificar el plan, ponerlo en marcha y evaluarlo, y así sucesivamente" (Murillo, F, 2010, 2011: 16). Logrando de esta manera un perfeccionamiento y dominio que le permiten al maestro producir conocimientos, innovar y transformar.

Se diseñó una encuesta dirigida a docentes y estudiantes, se aplicó una prueba diagnóstica de producción textual y para evaluar los textos se tuvo en cuenta rúbricas de evaluación con características del texto de acuerdo con su género. Se realizaron reflexiones permanentes y ajustes oportunos a través del diario de campo.

Se tomó como muestra el grado sexto dos, de la Institución Educativa Colegio La Salle de Ocaña Norte de Santander, conformado por 41 estudiantes, niños y niñas en edades entre los 11 y los 13 años de edad, donde un $25 \%$ son desplazados, pertenecientes a diversos tipos de familia

\section{Resultados}

Para la recolección de la información la Encuesta se aplicó a la totalidad de docentes de la sede secundaria, de igual forma fue aplicada a la totalidad de estudiantes que conforman el grupo sexto dos, población seleccionada para realizar la investigación, lo que garantiza la confiabilidad de los datos obtenidos

En la primera pregunta se pretendía describir con adjetivo los textos escritos por los estudiantes, ambos estamentos coincidieron en calificar los escritos como cortos. Con los alumnos se obtuvo 33 respuestas (81\%), 18 respuestas para los docentes, $(55 \%)$. Los maestros además agregaron la cualidad de confusos a los textos de los estudiantes con un total de 11 respuestas y un porcentaje del 33\% en esta apreciación. 
La segunda pregunta propuso una reflexión a la hora de escribir, se pudo comprobar cómo docentes y estudiantes exponen la problemática del escribir solo limitado al hecho de cumplir con un trabajo. Esto lo confirman 24 estudiantes $(58 \%)$ y 20 docentes que corresponde a un $61 \%$. Los niños y niñas no le dan importancia al texto de borrador pues esta característica obtuvo 0 respuestas y es ratificado con la respuesta de los docentes donde solo se observan 3 anotaciones en este inciso, para un porcentaje bajo del 9\%.,

En el proceso de escritura se destaca el papel orientador del docente, los alumnos frecuentemente recurren a ellos. Así lo afirmaron 33 alumnos, (80\%). Con lo anterior estuvieron de acuerdo 28 docentes, $85 \%$. Aunque 4 estudiantes, (10\%), dicen repasar el texto para corregirlo y otros 4 consultar el diccionario y diversos textos. Solo 2 docentes $(6 \%)$ mencionan que los alumnos realizan la primera tarea y solo un docente $(3 \%)$ evidencia el uso de diccionario o de textos para consultar.

La cuarta pregunta muestra la apreciación de 17 docentes, (52\%), sobre la idea que a los estudiantes no les gusta escribir; se presenta en oposición con lo que los educandos realmente expresan, ya que 27 alumnos, (66\%), manifiestan que les gusta mucho escribir.

Para finalizar la encuesta se elaboraron preguntas diferentes para alumnos y docentes pero encaminadas al mismo criterio. Los niños y niñas, 28 (68\%), ven como necesidad apremiante la inclusión de talleres de producción textual en las diversas áreas del conocimiento. Existe además la idea errónea de pensar que solo los ejercicios de escritura y producción textual son exclusivos al área de lengua castellana, así lo evidencio el total de 9 respuestas en lo que a esto se refiere, (22\%). Por su parte a los docentes se les preguntó si utilizaban estrategias pedagógicas que favorecieran la producción textual, obteniéndose un bajo porcentaje de quienes la utilizan frecuentemente $(18 \%)$. Generalmente los docentes que seleccionaron la respuesta anterior pertenecen al área de lengua castellana. Además en el momento de aplicar la encuesta los docentes dudaron en seleccionar la respuesta a esta pregunta y un alto porcentaje (67\%), solo se ubicó en el aspecto de algunas veces. Cabe destacar que cinco docentes fueron muy sinceros al señalar que nunca realizan ejercicios de producción textual en sus áreas, los cuales corresponden a un $15 \%$.

En la prueba diagnóstica de producción textual se aplicó una rejilla de evaluación que contenía las diversas características de un texto escrito. Estos fueron los resultados obtenidos:

En el proceso de escritura el total de los estudiantes $100 \%$ (41) no hace uso de un texto de borrador, elaboraron el texto en una hoja, la cual fue la misma que entregaron al docente. No se observó que realizaran un esquema o plan previo a la elaboración del texto. Pese a que la docente explicó e insistió en los aspectos y con relación a la adecuación comunicativa en los escritos de 15 estudiantes (37\%), se evidencia que no hay ningún intento de relacionar el escrito con lo que se le propone escribir. En el análisis de 17 escritos, (41\%) se observa 
que algo del escrito está relacionado con lo que se propone escribir, pueden o no presentarse características del texto solicitado (cuento). Por su parte solo en 9 estudiantes, es decir el 22\%, la mayor parte del escrito evidencia que sí está relacionado a lo que se propone escribir, aunque se divaga un poco en las características del texto solicitado.

Pasando a la coherencia en los escritos de 15 estudiantes, (37\%), no hay ningún intento de relacionar el cuento al tema asignado. Las ideas y escenas parecen estar ordenadas al azar. En 25 escritos, (61\%), se observa que está relacionado con el tema, aunque es un poco difícil de seguir. Las transiciones no son claras en más de una ocasión. Presentan algunas ideas repetidas, innecesarias o vacías de información. Solo en un estudiante se evidencia que la mayor parte del cuento está relacionado al tema y está bastante organizado, aunque una idea o escena parece fuera de lugar. No se encontró ningún escrito completamente relacionado al tema y bien organizado.

Analizando los aspectos de cohesión, en 38 estudiantes, (93\%), no se evidencian el uso de conectores, ni el uso de ningún signo de puntuación. En los textos de dos estudiantes hay de dos a tres errores en el uso de conectores. Se puede o no observar el uso del punto. Solo un estudiante presenta muy pocos errores, entre uno y dos, en el uso de conectores y se observa el uso de algunos signos de puntuación como la coma o el punto. No se encontraron textos con una cohesión adecuada a lo largo de todo el cuento.

Se observó que en la adecuación gramatical, el 100\% de los escritos presenta pocos errores gramaticales en el borrador final, es decir entre uno y dos errores. Como positivo se ve que de manera general los alumnos establecen relaciones adecuadas entre las diversas clases de palabras como género y número. Por otra parte en el léxico de 9 estudiantes, (22\%) hay más de cuatro errores semánticos. Hay 12 escritos que presentan de 3 a 4 errores, correspondiente a un 34\%. Solo 14 estudiantes, (29\%), presentó pocos errores semánticos, entre uno y dos errores. Solo 6 estudiantes, es decir un $15 \%$ no presentaron errores semánticos. Finalmente en relación a la ortografía un $83 \%$ de los estudiantes presenta más de cuatro errores de ortografía, esto corresponde a un número de 34 estudiantes, una cifra bastante alta. Se conoció de acuerdo a los diálogos establecidos con los niños y niñas que una de las fobias que se presentan a la hora de escribir es la excesiva corrección que hacen los docentes y que disgusta a los estudiantes. Sin embargo es necesario establecer y orientar hacia la necesidad de procurar un texto limpio al escribir.

Con base en el análisis obtenido de los resultados y del histórico de las pruebas saber se determinó la urgencia de implementar estrategias de producción textual para lo cual se diseñaron cuatro unidades didácticas en el área de ciencias sociales girando en torno a las diversas clases de texto, la primera como punto central en astronomía poética; en la segunda se utilizó el género narrativo a partir de la temática de la prehistoria e historia, 
la tercera se centró en textos descriptivos a partir de la construcción de un atlas geográfico y en la última se trabajó la elaboración de folletos a partir de los diversos proyectos transversales. En cada una de las estrategias generales se logró la participación efectiva del $100 \%$ de los estudiantes dando a conocer las evidencias del trabajo realizado a los diversos estamentos, como por ejemplo un libro de astronomía poética. La experiencia fue compartida por los demás becarios de la institución para establecer una propuesta conjunta de intervención institucional en producción textual en secundaria.

Se logró la creación de la escuela juvenil de escritores, donde el docente investigador se inscribe como formador, recibiendo orientación y permitiendo un pretexto más para escribir: el concurso regional del cuento. Donde se obtuvo la selección de cinco cuentos por el Ministerio de Educación Nacional para ser publicados en un libro. Se vinculó además a los padres de familia, a los estudiantes de once grado como críticos de los escritos.

Cabe destacar que el trabajo adelantado y los resultados obtenidos llevaron al docente investigador a proyectarse nacionalmente a través de la convocatoria de Fecode en el V concurso nota de maestros, donde obtuvo el primer puesto a nivel nacional y permitió a través de entrevistas concedidas para el programa encuentro y contrastes dar a conocer el desarrollo del proceso de producción textual en el área de ciencias sociales como una posibilidad para adelantar procesos de construcción de paz en el marco del postconflicto nacional.

\section{Conclusiones}

Se piensa que las competencias comunicativas son solo funciones específicas del área de lengua castellana. Después de aplicar esta investigación se comprende que es una herramienta para llevar con seguridad al aula y ser trabajada en cualquier área, logrando el desarrollo de competencias comunicativas. Partiendo principalmente de la motivación de los estudiantes.

En el proceso de evaluación es fundamental introducir procesos metacognitivos que permitan que el estudiante evidencie su propio proceso de aprendizaje. El verdadero papel del docente es lograr el desarrollo de las competencias en todo el grupo de estudiantes y debe tener claro que para lograrlo debe respetar los diversos ritmos de aprendizaje. Cuando el docente involucra a los padres de familia en los procesos del aula, haciendo ver la importancia de su papel, se logran mayores resultados. Es trascendental dejar de lado las quejas y reclamos para motivar a través de evidencias claras los logros alcanzados por pequeños que estos parezcan.

El contexto ofrece oportunidades que deben ser aprovechadas por el docente para proyectar el trabajo del aula. Por ejemplo la escuela de escritores fortalece la formación académica tanto de alumnos como de maestros, 
permitiendo la integración con otros grupos, la motivación por escribir para participar en los concursos establecidos, ofrece además la oportunidad de obtener resultados de evaluación y la posibilidad de acrecentar las competencias comunicativas de manera espontánea

Una de las principales fallas detectadas es la dificultad tanto de docentes como de alumnos en el manejo adecuado de diversas fuentes, estos procesos se deben fortalecer en las diversas áreas del conocimiento y de manera permanente hasta alcanzar un dominio de ellas.

El programa becas por la excelencia educativa fortaleció el proceso de formación de los docentes en el área de investigación haciendo consciente la necesidad de reflexionar, escribir, contar y trascender en diversos ámbitos para romper las barreras físicas de la escuela.

\section{Referencias}

Cassany, D. (1993). La cocina de la escritura. Barcelona: Anagrama.

Álvarez, T. \& Ramírez, R. (2006). Teorías o modelos de producción de textos en la enseñanza y el aprendizaje de la escritura. Universidad Complutense de Madrid y Universidad de Nariño Colombia. Recuperado el 3 de julio de 2016 de http://media.utp.edu.co/referencias-bibliograficas/uploads/referencias/articulo/33-teoraso-modelos-de-produccin-de-textos-en-laenseanza-y-el-aprendizaje-de-la-escriturapdf-snqQb-articulo.pdf

Avilés, S. (2012) La producción de textos en la escuela, una lectura desde el pensamiento complejo. Caso: secundaria José Martí. (Tesis de maestría en docencia y administración de la educación superior) Colegio de estudio de postgrados de Ciudad de México.

Recuperado el 4 de junio de 2016 de https://entremaestros.files.wordpress.com/2012/12/la-produccic3b3nde-textos-en-la-escuela.pdf

Chávez, Murata \& Uechara. (2012). Estudio descriptivo comparativo de la producción escrita descriptiva y narrativa de los niños de

quinto grado de educación primaria de las instituciones educativas de fe y Alegría del Perú, (Tesis de maestría) Pontificia

Universidad Católica en la escuela de postgrados. Lima Perú recuperado el 12 de marzo de 2016 de http://tesis.pucp.edu.pe/repositorio/bitstream/handle/123456789

/1704/CHAVEZ_MURATA_UEHARA_ESTUDIO_DESCRIPTIVO.pdf? sequence $=1$

Gómez, S. \& Álvarez, R. (2009) Competencias comunicativas en el área de ciencias sociales: un proyecto de extensión desde la universidad 
para la educación básica. Universidad industrial de Santander. Bucaramanga, Colombia. Recuperado el 5 de junio de 2016 http://www.humanas.unal.edu.co/iedu/files/2512/8618/3695/ComptComunicCS.pdf Ministerio de Educación Nacional-MEN. (2011).

Plan nacional de lectura y escritura de educación inicial, preescolar, básica y media. Bogotá, Colombia.

Recuperado el 5 de junio de 2016 http://www.colombiaaprende.edu.co/html/micrositios/1752/articles-317417_base_pnl.pdf

Murillo, F. (2010, 2011). Investigación Acción. Métodos en investigación especial. 3ra Educación especial.

Recuperado el 7 de junio de

2016 elhttps://www.uam.es/personal_pdi/stmaria/jmurillo/InvestigacionEE/Presentaciones/Curso_10

/Inv_accion_trabajo.pdf

(1).Citar este artículo como: Barbosa, J. \& Portillo, S. (2016). "Producir textos desde las ciencias sociales: una posibilidad de interdisciplinariedad". En: Revista La Tercera Orilla (17). Bucaramanga: Universidad Autónoma de Bucaramanga..

(2). Artículo arbitrado por Alicia Téllez. Magíster en Pedagogía. Universidad Industrial de Santander y Magíster en Evaluación en Educación. Universidad Santo Tomás. 